\title{
Characterizing an ensemble of interacting oscillators: The mean-field variability index
}

\author{
L. W. Sheppard, ${ }^{*}$ A. C. Hale, S. Petkoski, P. V. E. McClintock, and A. Stefanovska ${ }^{\dagger}$ \\ Department of Physics, Lancaster University, Lancaster LA1 4YB, United Kingdom
}

(Received 22 August 2012; published 9 January 2013)

\begin{abstract}
We introduce a way of characterizing an ensemble of interacting oscillators in terms of their mean-field variability index $\kappa$, a dimensionless parameter defined as the variance of the oscillators' mean field $r$ divided by the mean square of $r$. Based on the assumption that the overall mean field is the sum of a very large number of oscillators, each giving a small contribution to the total signal, we show that $\kappa$ depends on the mutual interactions between the oscillators, independently of their number or spectral properties. For purely random phasors, or a noninteracting ensemble of oscillators, $\kappa$ converges on 0.215 . Interactions push $\kappa$ in different directions: lower where there is interoscillator phase coherence, tending to zero for complete phase synchronization, or higher for amplitude synchronization or intermittent synchronization. We calculate $\kappa$ for several different cases to illustrate its utility, using both numerically simulated data and electroencephalograph signals from the brains of human subjects while awake, while anesthetized, and while undergoing an epileptic fit.
\end{abstract}

DOI: 10.1103/PhysRevE.87.012905

PACS number(s): 05.45.Tp, 89.75.Fb, 89.70.-a

\section{INTRODUCTION}

There exist in nature examples of cooperative systems composed of a large number of units that mutually interact and can exhibit a range of synchronization phenomena. The neurons in the human brain are an obvious case in point [1] but, quite generally, communication at the intercellular level [2] and between subcellular components [3] is crucial to the development and function of all multicellular organisms. Much progress has been made in studying the associated phenomena by investigating idealized ensembles of coupled oscillators, as in the Kuramoto model [4]. General overviews of Kuramoto oscillator ensembles have been provided by Acebrón et al. [5] and by Strogatz [6]. Their use in modeling brain activity in particular is described in Refs. [7-11].

In the case of the brain, interactions and synchronization can occur on many different levels [12], ranging from the cellular [8] up to the macroscopic where the huge groups of neurons that are responsible for the different electroencephalograph (EEG) waves observed on the scalp can themselves interact as distinct entities both with each other [13,14] and with some of the oscillatory processes of the cardiovascular system [15]. In what follows we will be primarily concerned with low-level interactions between the basic oscillators, thinking particularly of the neurons in the brain.

Although Kuramoto models can often replicate observed phenomena at a qualitative level, it is usually difficult to make detailed comparisons with reality because relatively few parameters of a real system are in practice accessible for direct measurement. In particular the type and strength of coupling between units may be difficult to determine. Sometimes the characteristics of an individual oscillator, and the number of oscillators, may be undetermined. In the case of an EEG measurement, the collective behavior of billions of neurons may be reduced to a univariate voltage time series [16]. Although complex nonlinear methods have been introduced

\footnotetext{
*Present address: Division of Biology, Imperial College London, Silwood Park Campus, Ascot SL5 7PY, United Kingdom.

†aneta@lancaster.ac.uk
}

for the study of neuronal ensembles, relevant information can also be inferred from relatively simple statistical measures such as the variance of an EEG signal power [17].

Recent work on more complex oscillator models has produced examples of oscillator ensembles exhibiting partial synchrony [18], multistable synchronous dynamics [19], intermittent synchrony [20], synchronous subensembles [21], and time variations of their parameters [22]. Temirbayev et al. [23] describe some parameters of the ensemble that change as the oscillators synchronize. We are motivated to seek parameters of an oscillator ensemble system that depend on its synchronization state, independent of the details of the oscillators.

In this paper we will show that, by examining the fluctuations in the strength of the mean field, we can infer the type of interaction occurring between the oscillators, even without knowing their number or type. We characterize the interactions through the introduction of a measure, the mean-field variability index (MFVI) $\kappa$, which we introduce in Sec. II. In Sec. III we discuss $\kappa$ for the case when there are no interactions between the oscillators. The main part of the paper, which relates to the more interesting case where there are nonzero interoscillator interactions, and therefore some degree of coherence, is presented in Sec. IV, considering $\kappa$ for several different scenarios. Some special cases (coherent cases with higher harmonics, complex coherence, correlated amplitudes, and intermittency) are analyzed in Sec. V. Section VI reveals distinct differences in the $\kappa$ values obtained from analysis of EEG data under different conditions (wakefulness, anesthesia, and epilepsy), thus demonstrating the potential usefulness of the MFVI. Finally, in Sec. VII we summarize and draw conclusions.

\section{THE MEAN FIELD OF AN ENSEMBLE AND THE MEAN-FIELD VARIABILITY INDEX $\kappa$}

Our approach involves investigation of the distribution of amplitude values for a signal composed of many oscillatory components. In the case of real signals the overall amplitude can be determined by means of a Hilbert transform or considered as the magnitude of a wavelet transform component, or 
found in other ways [24]. Analytically, and in our oscillator ensemble models, we calculate the magnitude of the mean field.

Consider an ensemble of $N$ sinusoidal oscillators represented as complex waveforms $w_{i}=a_{i}(t) e^{i \phi_{i}(t)}$, with a mean field $F(t)=r(t) e^{i \Phi(t)}$, where at each instant in time

$$
F=\frac{1}{N} \sum_{i=1}^{N} w_{i}
$$

Note that in this representation the field is an average, normalized by $\frac{1}{N}$ for ease of working. The results below follow for all large $N$ and can thus be applied to the total field where $N$ is unknown. Both the amplitude and rate of phase growth of the oscillators may vary. We can express the magnitude of the mean field as

$$
r=\sqrt{\left(\frac{1}{N} \sum_{i=1}^{N} w_{i}\right)\left(\frac{1}{N} \sum_{j=1}^{N} w_{j}^{*}\right)}
$$

This is the square root of the sum of a Hermitian matrix of terms. Note that $r$ is real by definition. The terms under the radical can be broken into diagonal and off-diagonal parts

$$
r=\frac{1}{N} \sqrt{\sum_{i=1}^{N} w_{i} w_{i}^{*}+2 \operatorname{Re} \sum_{i=1}^{N-1} \sum_{j=i+1}^{N} w_{i} w_{j}^{*}}
$$

where the asterisk denotes the complex conjugate. We define

$$
\kappa=\frac{E\left(r^{2}\right)-E(r)^{2}}{E\left(r^{2}\right)},
$$

where $E()$ is the expectation value, or average over possibilities (the expectation value is the same for all time assuming stationarity). We will also use angular brackets to indicate expectation values.

Evaluated by a series of complex measurements in time $T$, each oscillator has a power $p_{i}=\frac{1}{T} \sum_{t=1}^{T} w_{i}(t) w_{i}(t)^{*}$ and the sum of all the powers is $P=\sum_{i=1}^{N} p_{i}$. Assuming that over time the system explores the space of all possible states, we can estimate $E\left(r^{2}\right)$ by finding the power and $E\left(r^{2}\right)-E(r)^{2}$ by finding the variance of the mean-field amplitude.

We will consider three types of synchronization.

(i) In frequency synchronization the oscillators have similar spectral characteristics but do not share phase or amplitude information over time. It may arise as a result of coupling between oscillators or on account of nonautonomous influences. Although not true synchronization, it can result in the formation of clear peaks in the power spectrum of the mean field and phase differences between oscillators that grow exceedingly slowly.

(ii) In phase synchronization the oscillators tend to preserve a fixed phase difference (equal to zero for a homogeneous ensemble) [25] despite the frequency variability that can arise in some cases [26].

(iii) In amplitude synchronization the amplitude variations of the oscillators are correlated.

In reality these phenomena are often combined with each other, as well as with external sources of noise, and they may also be intermittent. Note also that in the real world, frequency synchronization and coupling of phase or amplitude in the time domain can result from interactions between individual members of the ensemble and some third party influencing all their frequencies, phases, or amplitudes together [27].

\section{THE NONINTERACTING CASE $\boldsymbol{\kappa}=\mathbf{0 . 2 1 5}$}

We first examine the case where there are no interactions between the oscillators. Consider a mean-field signal as arising from the combination of a large number of complex oscillatory signals, each making a small contribution to the ensemble, and having uncorrelated phases and amplitudes

$$
r(t) e^{i \Phi(t)}=\frac{1}{N} \sum_{i=1}^{N} a_{i}(t) e^{i \phi_{i}(t)}=\frac{1}{N} \sum_{i=1}^{N}\left[x_{i}(t)+i y_{i}(t)\right] .
$$

If the ensemble is composed of many noninteracting oscillators, then the sum of all their values $\sum_{i=1}^{N} w_{i}(t)$ at any given time is a sum of many independent random variables. Hence $\sum_{i=1}^{N} x_{i}(t)$ and $\sum_{i=1}^{N} y_{i}(t)$ both belong to independent Gaussian distributions with zero mean and width $\sigma$. It then follows that the magnitude of $\sum_{i=1}^{N}\left[x_{i}(t)+i y_{i}(t)\right]$ is described by a Rayleigh distribution [28] and the phase is given by a uniform distribution over the interval $[-\pi, \pi]$.

Using normalized distributions, it follows that

$$
E(r)=\int_{0}^{\infty} r \frac{r}{\sigma^{2}} e^{-r^{2} / 2 \sigma^{2}} d r=\sigma \sqrt{\frac{\pi}{2}}
$$

and

$$
E\left(r^{2}\right)=\int_{0}^{\infty} r^{2} \frac{r}{\sigma^{2}} e^{-r^{2} / 2 \sigma^{2}} d r=2 \sigma^{2}
$$

Hence a complex Gaussian white noise signal-the maximally desynchronized case_-gives

$$
\kappa=1-\frac{\pi}{4} \approx 0.215,
$$

a value that is independent of both the number and amplitudes of the oscillators that produce the signal.

Note that this case also includes ensembles exhibiting frequency synchronization, where the oscillators have similar spectral characteristics but without any phase or amplitude information shared over time. To determine $\kappa$ correctly, the period of measurement must of course be sufficiently long, or the signal sufficiently broadband, to obtain many independent measurements of $r$.

\section{THE COHERENT CASE $\boldsymbol{\kappa}<0.215$}

Oscillators can become phase synchronized by coupling. It is clear that if all the oscillators have become phase locked, the only variability in the mean field must come from amplitude variability: If there is no amplitude variability then $\kappa=0$ because the mean-field amplitude is fixed. We consider two kinds of partial phase synchronization producing $0<\kappa<0.215$. The first kind involves some of the oscillators becoming fully coherent while others remain unsynchronized; the second involves all of the oscillators becoming partially coherent with a mean coherence $c$. 
Squaring Eq. (3) we obtain

$$
r^{2}=\frac{1}{N^{2}}\left[\sum_{i=1}^{N} w_{i} w_{i}^{*}+2 \sum_{i=1}^{N-1} \operatorname{Re}\left(\sum_{j=i+1}^{N} w_{i} w_{j}^{*}\right)\right] .
$$

For unrelated phase and amplitude data, including ensembles of frequency-synchronized broadband oscillators

$$
\left\langle 2 \sum_{i=1}^{N-1} \operatorname{Re}\left(\sum_{j=i+1}^{N} w_{i} w_{j}^{*}\right)\right\rangle=0,
$$

so that

$$
E\left(r^{2}\right)=\frac{P}{N^{2}} .
$$

For an ensemble of unit phasors (each of which is a complex number whose magnitude is normalized to unity and whose argument represents the phase of the oscillation) this reduces to $1 / N$.

We represent the $i$ th oscillator by $w_{i}=a_{i} e^{i \phi_{i}}$, thus having an amplitude $a_{i}$ and a phase $\phi_{i}$ at every moment in time. Where phase relationships are maintained between oscillators the mean field is no longer a random walk and the expectation value of $c_{i j}=e^{i \phi_{i}} e^{-i \phi_{j}}$ is nonzero. We define $C_{i j}$ to be $\left\langle e^{i \phi_{i}} e^{-i \phi_{j}}\right\rangle$. The phase coherence [29] of $w_{i}$ and $w_{j}$ is equal to the magnitude of $C_{i j}$.

We will illustrate various cases using a classic homogeneous Kuramoto model augmented by noise,

$$
\begin{aligned}
\dot{\phi}_{k}(t) & =\omega_{k}+\frac{\varepsilon}{N} \sum_{j=1}^{N} \sin \left[\phi_{j}(t)-\phi_{k}(t)\right]+\eta_{k}(t), \\
k & =1, \ldots, N .
\end{aligned}
$$

Here $\phi_{k}$ is the phase of the $k$ th oscillator at time $t$ and $\omega_{k}$ is its natural frequency or eigenfrequency, chosen at random from a symmetric unimodal distribution $g(\omega)$ whose width is characterized by a parameter $\gamma$; we define the mean of $g(\omega)$ to be zero by transforming to the rotating frame. There exists all-to-all coupling between the $N$ nodes with strength $\varepsilon \in \mathbb{R}^{+}$. The noise $\eta_{k}(t)$ (if applied) follows a Gaussian distribution with zero mean and intensity $2 D$. Initially at time $t=0$ all the phases are randomly selected from a uniform distribution $[0, \ldots, 2 \pi)$. The collective rhythm is quantified by

$$
r(t) e^{i \Phi(t)}=\frac{1}{N} \sum_{j=1}^{N} e^{i \phi_{j}(t)},
$$

where $\Phi(t)$ is the average phase and $r(t)$ is the mean-field amplitude, which also describes the synchronization: $r=$ 0 for fully incoherent oscillators and $r=1$ for the case of complete synchronization when all the oscillators have identical phases. The value of the mean field depends only on the interplay between the coupling strength and the diversity in the ensemble, described either by the width of the natural distribution of frequencies $\gamma$ or by the noise intensity $D$. Hence there exists a critical value of the coupling $\varepsilon_{c}$ above which some of the oscillators become coherent, i.e., phase locked with constant phase difference, resulting in a nonzero mean field. For increasing coupling, the number of locked oscillators increases, while the phase differences between the locked oscillators decreases, and in the infinite limit all of the oscillators become locked to the same mean phase. When required all $\omega_{k}$ are set to zero to obtain a homogeneous ensemble. However, for finite-size ensembles $N<\infty$, the mean-field value is fluctuating with a variance of order $1 / N$ and for the noncoherent case $\varepsilon<\varepsilon_{c}$ the size of the mean field is in the range of $O\left(N^{-1 / 2}\right)$ [5]. This is of crucial importance for our analysis of real systems, where finite-size effects are inevitable.

The white noise case was verified numerically with a Kuramoto ensemble of 1000 desynchronized oscillators [Eq. (13)] for $\omega_{k}=0 \forall k, D=0.2$. A coupling of $\varepsilon=0.01$ (well below the critical coupling) yielded $\kappa=0.2097$ with standard deviation 0.0123 for 1 -min segments from a 20 -min time series.

\section{A. Some oscillators coherent}

Consider the case where the coupling between oscillators is sufficient to fully synchronize some of them, but not others. Let $c_{i j}=1$ for all $i, j<p$, the total number of synchronized oscillators. A further $q$ oscillators remain unsynchronized. We assume that the phases of the synchronized oscillators are all the same and the phases of the unsynchronized oscillators are uniformly distributed. Note, however, that this will not be exactly true in the case of the Kuramoto model (where the phases of the synchronized oscillators, though locked, are not all the same and the phases of the unsynchronized oscillators are not uniformly distributed). We express all phases relative to the phase of the synchronized oscillators, which is set to zero.

If phase relationships are independent of amplitude relationships,

$$
\begin{aligned}
E\left(r^{2}\right)= & \frac{1}{N^{2}}\left(P+2 \sum_{i=2}^{p} \sum_{j=1}^{i}\left\langle a_{i} a_{j}\right\rangle+2 \operatorname{Re} \sum_{i=2}^{p} \sum_{j=p+1}^{p+q}\left\langle a_{i} a_{j}\right\rangle e^{i \phi_{j}}\right. \\
& \left.+2 \operatorname{Re} \sum_{i=p+2}^{p+q} \sum_{j=1}^{i}\left\langle a_{i} a_{j}\right\rangle c_{i j}\right) .
\end{aligned}
$$

If amplitude variability is also itself uncorrelated,

$$
\begin{aligned}
E\left(r^{2}\right)= & \frac{1}{N^{2}}\left(P+2 \sum_{i=2}^{p} \sum_{j=1}^{i}\left\langle a_{i}\right\rangle\left\langle a_{j}\right\rangle\right. \\
& +2 \operatorname{Re} \sum_{i=2}^{p} \sum_{j=p+1}^{p+q}\left\langle a_{i}\right\rangle\left\langle a_{j}\right\rangle e^{i \phi_{j}} \\
& \left.+2 \operatorname{Re} \sum_{i=p+2}^{p+q} \sum_{j=1}^{i}\left\langle a_{i}\right\rangle\left\langle a_{j}\right\rangle c_{i j}\right) .
\end{aligned}
$$

For a population of oscillators without amplitude correlations but with the same mean amplitude, $\left\langle a_{i}\right\rangle=a$, so

$$
\begin{aligned}
E\left(r^{2}\right)= & \frac{1}{N^{2}}\left(P+2 \sum_{i=2}^{p} \sum_{j=1}^{i} a^{2}+2 \operatorname{Re} \sum_{i=2}^{p} \sum_{j=p+1}^{p+q} a^{2} e^{i \phi_{j}}\right. \\
& \left.+2 \operatorname{Re} \sum_{i=p+2}^{p+q} \sum_{j=1}^{i} a^{2} c_{i j}\right) .
\end{aligned}
$$


To evaluate $E(r)$, Eq. (3) becomes [taking out a factor of $\left.\sqrt{P+p(p-1) a^{2}}\right]$

$$
\begin{aligned}
E(r)= & \frac{1}{N}\left\langle\sqrt { ( P + p ( p - 1 ) a ^ { 2 } ) } \left[ 1+\frac{1}{\left(P+p(p-1) a^{2}\right)}\right.\right. \\
& \times\left(2 \operatorname{Re} \sum_{i=2}^{p} \sum_{j=p+1}^{p+q} a^{2} e^{i \phi_{j}}\right. \\
& \left.\left.\left.+2 \operatorname{Re} \sum_{i=p+2}^{p+q} \sum_{j=1}^{i} a^{2} c_{i j}\right)\right]^{1 / 2}\right\rangle .
\end{aligned}
$$

We evaluate $E(r)$ as a Taylor expansion, setting

$$
\begin{aligned}
x= & \frac{1}{P+p(p-1) a^{2}} \\
& \times\left(2 \operatorname{Re} \sum_{i=2}^{p} \sum_{j=p+1}^{p+q} a^{2} e^{i \phi_{j}}+2 \operatorname{Re} \sum_{i=p+2}^{p+q} \sum_{j=1}^{i} a^{2} c_{i j}\right),
\end{aligned}
$$

assuming that $|x|<1$ and the quantity under the radical (the contribution from self terms and the coherent part of the ensemble) dominates the mean field. As a sum of random phasors, $\langle x\rangle=0$, giving

$$
E(r) \approx \frac{1}{N} \sqrt{P+p(p-1) a^{2}}\left\langle 1-\frac{1}{8} x^{2} \cdots\right\rangle,
$$

and so Eq. (4) reduces to

$$
\kappa \approx 1-\left(1-\frac{1}{8}\left\langle x^{2}\right\rangle\right)^{2} .
$$

We must evaluate

$$
\begin{aligned}
\left\langle x^{2}\right\rangle= & \frac{1}{\left[P+p(p-1) a^{2}\right]^{2}} \\
& \times\left\langle\left(2 \operatorname{Re} \sum_{i=2}^{p} \sum_{j=p+1}^{p+q} a^{2} e^{i \phi_{j}}+2 \operatorname{Re} \sum_{i=p+2}^{p+q} \sum_{j=1}^{i} a^{2} c_{i j}\right)\right. \\
& \left.\times\left(2 \operatorname{Re} \sum_{k=2}^{p} \sum_{l=p+1}^{p+q} a^{2} e^{i \phi_{l}}+2 \operatorname{Re} \sum_{k=p+2}^{p+q} \sum_{l=1}^{i} a^{2} c_{k l}\right)\right\rangle .
\end{aligned}
$$

Using the fact that all except self terms have an expectation value of zero, we obtain

$$
\left\langle x^{2}\right\rangle=\frac{a^{4}}{\left[P+p(p-1) a^{2}\right]^{2}}\left[2 p^{2} q+q(q-1)\right] .
$$

For the canonical Kuramoto model, composed of individual phase oscillators in the absence of noise, with a distribution of frequencies and coupling greater than the critical value required to synchronize some of the oscillators,

$$
\kappa \approx \frac{1}{4} \frac{1}{\left(p^{2}+q\right)^{2}}\left[2 p^{2} q+q(q-1)\right]
$$

If we take $p \approx N \bar{r}$ and $q \approx N(1-\bar{r})$, where $\bar{r}$ is the measured mean field of a partially synchronized $N$-oscillator Kuramoto ensemble, we expect

$$
\begin{aligned}
\kappa \approx & \frac{1}{4}\left(\frac{1}{\left[N^{2} \bar{r}^{2}+N(1-\bar{r})\right]^{2}}\right) \\
& \times\left\{2 N^{3} \bar{r}^{2}(1-\bar{r})+N(1-\bar{r})[N(1-\bar{r})-1]\right\},
\end{aligned}
$$

or to leading order in $N$

$$
\kappa \approx \frac{1}{2} \frac{1}{N \bar{r}^{2}}(1-\bar{r})
$$

We have tested this estimate by running numerical simulations. We set up a nonhomogeneous ensemble of 50000 Kuramoto oscillators with $\gamma=0.5$ and $D=0$, evolving over $500 \mathrm{~s}$ with a time step of $0.05 \mathrm{~s}$. The transient in the first $200 \mathrm{~s}$ was discarded to leave $300 \mathrm{~s}$ of data. Each result is averaged over 25 runs. We find $\varepsilon_{c}=2 \gamma$, and below $\varepsilon_{c}$ incoherent behavior is observed. For high values of the coupling $\varepsilon$ a large proportion of the oscillators become synchronized and $r$ approaches unity.

In Fig. 1(a) we see the manner in which $\kappa$ falls off with respect to coupling: With low coupling $\kappa \sim 0.215$ (incoherent case) and it falls towards zero for higher coupling (high degree of coherence). In Fig. 1(b) we present the predicted $\kappa$ values for this idealized picture, alongside values generated by actually picking $r N$ phasors with the same phase and $(1-r) N$ phasors from a uniform distribution over the interval $[-\pi, \pi]$ (average of 1000 realizations for each $r$ ). We use these ideal phasors
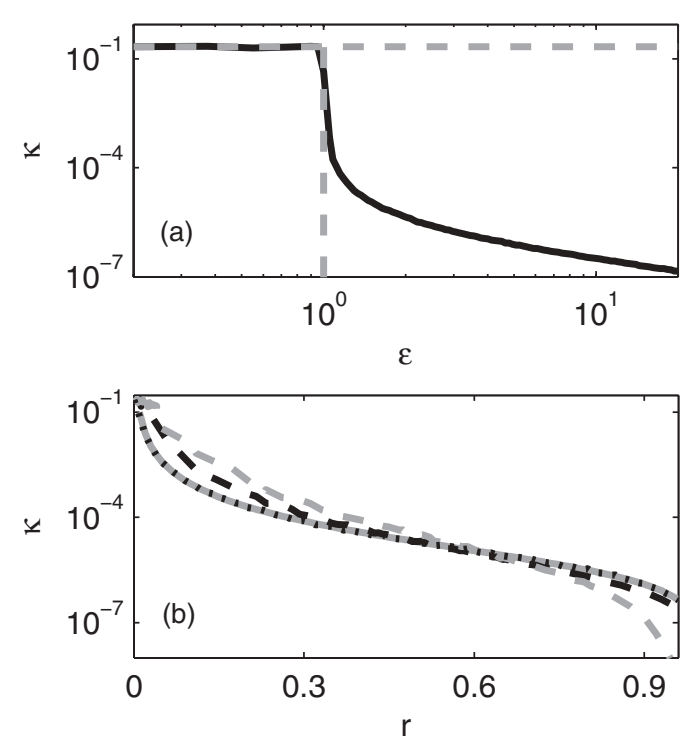

FIG. 1. (a) Relationship between $\kappa$ and the coupling constant $\varepsilon$ (black solid line) for the classic Kuramoto model with a Lorentzian distribution of frequencies, where $\gamma=0.5$ and $N=50000$. The horizontal dashed gray line indicates $\kappa$ for white noise and the vertical dashed gray line indicates $\varepsilon_{c}$. (b) Relationship between $\kappa$ and $r(N$ fixed) for the classic Kuramoto model with Lorentzian (black dashed line) and Gaussian (gray dashed line) distributions of frequencies with a proportion of the oscillators synchronized. A simulation of ideal phasors (black dotted line) is almost coincident with the expected $\kappa$ values (gray solid line) calculated directly from Eq. (24). These values differ slightly from those for Kuramoto oscillators with a Lorentzian distribution, over a range of several powers of 10 in $\kappa$. 
to represent the state of a set of oscillators in which those oscillators that are not phase locked together have phases completely independent of the rest. The approximation (24) is accurate. However, in any real ensemble the phase coherence of the synchronized oscillators is slightly less than unity and the phase distribution of the unsynchronized oscillators is not entirely uniform.

In Fig. 1(b) we also present a simulation in which the distribution of oscillator natural frequencies is Gaussian rather than Lorentzian. We see that the difference between the idealized model (of some oscillators fully synchronized and others fully desynchronized) and the actual results depends on the details of the simulation used.

\section{B. All oscillators partially coherent}

In the case of a homogeneous population of unit oscillators maintaining zero phase difference on average, $C_{i j}=\left\langle c_{i j}\right\rangle$ is expected to be real and positive and thus equal to the phase coherence. Of course, $C_{i i}$ is always equal to unity. We define the mean coherence $c=\left\langle\frac{1}{N(N-1)} \sum_{i=1}^{N-1} \sum_{j=i+1}^{N}\left(c_{i j}+c_{i j}^{*}\right)\right\rangle$ to obtain

$$
E\left(r^{2}\right)=\frac{1}{N^{2}}\left(P+\sum_{i=1}^{N-1} \sum_{j=i+1}^{N}\left\langle a_{i} a_{j} c_{i j}+a_{i} a_{j} c_{i j}^{*}\right\rangle\right)=\frac{1}{N^{2}}\left(P+2 \sum_{i=1}^{N-1} \sum_{j=i+1}^{N}\left\langle a_{i} a_{j}\right\rangle c\right)
$$

if phase relationships are independent of amplitude relationships. If the amplitude variability is also itself uncorrelated, then

$$
E\left(r^{2}\right)=\frac{1}{N^{2}}\left(P+2 \sum_{i=1}^{N-1} \sum_{j=i+1}^{N}\left\langle a_{i}\right\rangle\left\langle a_{j}\right\rangle c\right) .
$$

For a population of oscillators without amplitude correlations but with the same mean amplitude, $\left\langle a_{i}\right\rangle=a$ and so

$$
E\left(r^{2}\right)=\frac{1}{N^{2}}\left[P+N(N-1) a^{2} c\right]
$$

To evaluate $E(r)$, Eq. (3) becomes

$$
r=\frac{1}{N}\left[\left(P+N(N-1) a^{2} c\right)+\left(\sum_{\substack { i=1 \\
\begin{subarray}{c}{j=1 \\
j \neq i{ i = 1 \\
\begin{subarray} { c } { j = 1 \\
j \neq i } }\end{subarray}}^{N}\left[a_{i} a_{j}\left(c_{i j}-c\right)\right]\right)\right]^{1 / 2} .
$$

Taking out a factor of $\sqrt{P+N(N-1) a^{2} c}$, we obtain

$$
E(r)=\frac{1}{N}\left\langle\sqrt{P+N(N-1) a^{2} c}\left[1+\frac{1}{\left(P+N(N-1) a^{2} c\right)}\left(\sum_{i=1}^{N} \sum_{\substack{j=1 \\ j \neq i}}^{N}\left[a_{i} a_{j}\left(c_{i j}-c\right)\right]\right]^{1 / 2}\right) .\right.
$$

We evaluate $E(r)$ as a Taylor expansion, setting

$$
x=\frac{1}{P+N(N-1) a^{2} c}\left(\sum_{\substack { i=1 \\
\begin{subarray}{c}{j=1 \\
j \neq i{ i = 1 \\
\begin{subarray} { c } { j = 1 \\
j \neq i } }\end{subarray}}^{N}\left[a_{i} a_{j}\left(c_{i j}-c\right)\right]\right)
$$

and assuming that $|x|<1$, i.e., that the sum of all $\left(c_{i, j}-c\right) / c$ is a small fraction when the ensemble is fluctuating about a steady state. By construction $\langle x\rangle=0$, giving

$$
E(r) \approx \frac{1}{N} \sqrt{P+N(N-1) a^{2} c}\left(1-\frac{1}{8} x^{2} \cdots\right\rangle
$$

and as before

$$
\kappa \approx 1-\left(1-\frac{1}{8}\left\langle x^{2}\right\rangle\right)^{2}
$$


We must examine

$$
\left\langle x^{2}\right\rangle=\frac{1}{\left[P+N(N-1) a^{2} c\right]^{2}}\left\langle\left(\sum_{i=1}^{N} \sum_{\substack{j=1 \\ j \neq i}}^{N}\left[a_{i} a_{j}\left(c_{i j}-c\right)\right] \sum_{k=1}^{N} \sum_{\substack{l=1 \\ l \neq k}}^{N}\left[a_{k} a_{l}\left(c_{k l}-c\right)\right]\right)\right\rangle,
$$

where there is a total of $[N(N-1)]^{2}$ terms in the numerator. The denominator also grows as $N^{4}$.

The $N^{2}(N-1)^{2}$ different

$$
a_{i} a_{j} a_{k} a_{l}\left(c_{i j} c_{k l}-c c_{i j}-c c_{k l}+c^{2}\right)
$$

terms have different expectation values depending on whether any of the oscillator indices is repeated. The most obvious special cases are the $N(N-1)$ self terms for which $i=k$ and $j=l$, or the $N(N-1)$ for which $i=l$ and $j=k$, in which case

$$
\begin{aligned}
\left\langle c_{i j} c_{j i}-c c_{i j}-c c_{j i}+c^{2}\right\rangle & =\left\langle c_{i j} c_{i j}^{*}-c c_{i j}-c c_{i j}^{*}+c^{2}\right\rangle \\
& =1-c^{2} .
\end{aligned}
$$

We also need to know how many terms contain $i=l, i=k$, $j=l$, or $j=k$, how many terms are of the form $i \neq j \neq k \neq$ $l$, and which group of terms dominates the numerator of $\left\langle x^{2}\right\rangle$.

We consider the population of oscillators to be homogeneous if $\left\langle c_{i j} c_{k l}\right\rangle=c^{2}$ for $i, j, k, l$ are all different. In this case most terms cancel and the leading-order term in the numerator of $\left\langle x^{2}\right\rangle$ is of order $N^{3}$ (see below). If for large $N$ there exist distinct groups of oscillator indices for which $\left\langle c_{i j} c_{k l}\right\rangle>c^{2}$ when $i, j, k, l \varepsilon G_{m}$, with these groups making up a significant proportion of the total number of oscillators, then we consider the population to be inhomogeneous.

\section{The homogeneous case}

There are $N(N-1)$ terms of the form

$$
a_{i} a_{j} a_{i} a_{j}\left(c_{i j} c_{i j}-c c_{i j}-c c_{i j}+c^{2}\right),
$$

$N(N-1)$ terms of the form

$$
a_{i} a_{j} a_{j} a_{i}\left(c_{i j} c_{j i}-c c_{i j}-c c_{j i}+c^{2}\right),
$$

and $N(N-1)(N-2)$ terms of the form

$$
a_{i} a_{j} a_{k} a_{j}\left(c_{i j} c_{k j}-c c_{i j}-c c_{k j}+c^{2}\right)
$$

(i.e., where $j=l$ ), and the same number for $i=k, i=l$, or $j=k$. There remain $N(N-1)(N-2)(N-3)$ terms of the form

$$
a_{i} a_{j} a_{k} a_{l}\left(c_{i j} c_{k l}-c c_{i j}-c c_{k l}+c^{2}\right),
$$

where $i \neq j \neq k \neq l$. For a population without amplitude correlations,

$$
\left\langle a_{i} a_{j} a_{k} a_{l}\right\rangle=\left\langle a_{i}\right\rangle\left\langle a_{j}\right\rangle\left\langle a_{k}\right\rangle\left\langle a_{l}\right\rangle,
$$

where $i \neq j \neq k \neq l$, whereas

$$
\begin{aligned}
\left\langle a_{i}^{2} a_{j}^{2}\right\rangle & =\left\langle a_{i}^{2}\right\rangle\left\langle a_{j}^{2}\right\rangle=p_{i} p_{j}, \\
\left\langle a_{i} a_{j}^{2} a_{k}\right\rangle & =\left\langle a_{i}\right\rangle\left\langle a_{j}^{2}\right\rangle\left\langle a_{k}\right\rangle=\left\langle a_{i}\right\rangle p_{j}\left\langle a_{k}\right\rangle,
\end{aligned}
$$

and so on.
In Fig. 2(a) we see the dependence on $N$ of the numbers of the different types of terms. For large $N$ the $i \neq j \neq$ $k \neq l$ terms are most numerous, however for these terms $\left\langle c_{i j} c_{k l}\right\rangle-c^{2}=0$. Only terms where two or more indices are equal contribute to $\left\langle x^{2}\right\rangle$, assuming that there are no amplitude correlations.

The expectation values $\left\langle c_{i j} c_{i l}\right\rangle$, etc., will depend on the details of the ensemble, but we can see that altogether there
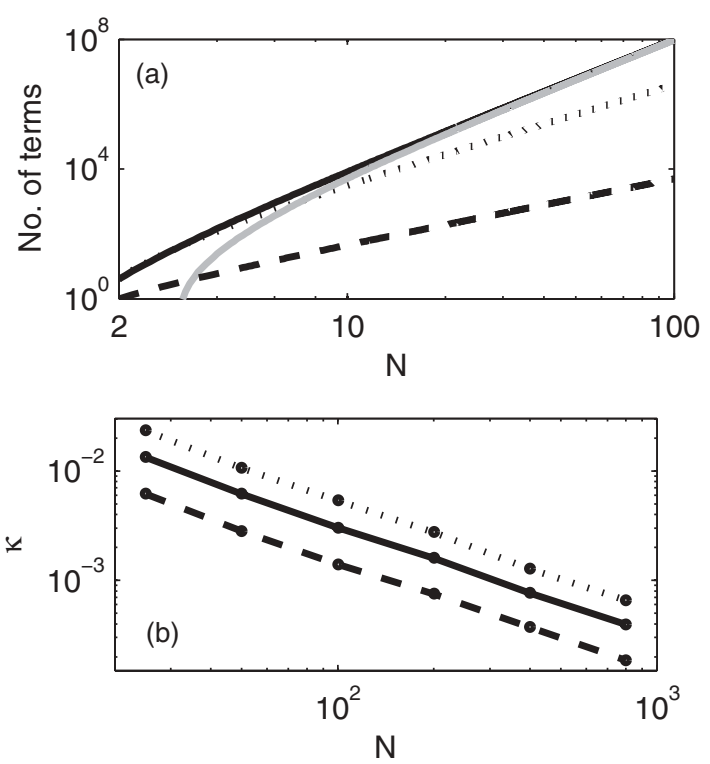

FIG. 2. (a) Number of terms of different types occurring in the numerator of $\left\langle x^{2}\right\rangle$ in Eq. (32) as functions of $N$. The number of self terms $i=k$ and $j=l$, or $i=l$ and $j=k$, increases as $N^{2}$ (dashed black line). The number of $i=l, i=k, j=l$, or $j=k$ terms increases as $N^{3}$ (dotted black line) and the number of $i \neq j \neq$ $k \neq l$ terms increases as $N(N-1)(N-2)(N-3)$ (gray line), i.e., approximately as $N^{4}$ for large $N$. Thus the total number of terms in the numerator increases as $N^{4}$ (solid black line), as does the denominator of $\left\langle x^{2}\right\rangle$. However, in the absence of amplitude correlations, only the $i=l$ or $k$ and $j=l$ or $k$ terms produce significant net contributions to the numerator of $\left\langle x^{2}\right\rangle$, which falls off as $1 / N$. (b) Dependence of $\kappa$ on $N$ for noisy homogeneous Kuramoto ensembles $\left(\omega_{k}=0\right)$ drawn from three different distributions (different $c$ and $\left\langle c_{i j} c_{k j}\right\rangle$ ). The lowest coherence (smallest coupling $\varepsilon=5.0$ ) case is shown at the top (dotted line), the highest coherence (largest coupling $\varepsilon=6.5$ ) at the bottom (dashed line), and an intermediate case in the middle (solid line). In each case the points are calculated from numerical simulations of Eq. (13) and the lines connecting them are guides to the eye. The gradient of the log-log plots is -1 , indicating the inverse relationship. 
are $4 N(N-1)(N-2)$ terms for which $i=k, j=l, i=l$, or $j=k$, plus $2 N(N-1)$ terms when $i=k$ and $j=l$, or $i=l$ and $j=k$. The order $N^{3}$ contribution dominates the numerator of $\left\langle x^{2}\right\rangle$.

When $c>0$, the $[N+N(N-1) c]^{2}$ factor in the denominator of Eq. (32) has an $N^{4}$ dependence and thus $\left\langle x^{2}\right\rangle$ tends towards zero as $1 / N$ for large $N$. Even if $c$ is small in absolute terms, if $N$ is large enough then the mean field will always be dominated by the directed walk in the complex plane resulting from the agreement on a single mean phase at each point in time.

Plugging $\left\langle x^{2}\right\rangle$ back into Eq. (30) we see that, for large $N$, both the root-mean-square field and $E(r)$ converge on $\mathrm{Na}$ times the square root of $c$, the mean phase coherence of a pair of oscillators in the ensemble. Hence the mean field of all the oscillators is proportional to the square root of the mean phase coherence of one pair of oscillators.

The limiting case is complete phase coherence between all oscillators in the ensemble, $c=1, E(r)=E\left(r^{2}\right)$, in which case the variance of the mean-field magnitude is zero, as is $\kappa$. We can verify that

$$
\kappa \approx \frac{1}{4}\left\langle x^{2}\right\rangle
$$

then depends on $1 / N$ for large $N$. In our classic homogeneous Kuramoto model augmented by the noise term, we use Eq. (13) and choose $\omega_{k}=0 \forall k$. The Gaussian white noise term amplitude is set at $D=0.2$. The integration scheme is backward finite differencing with an integration time step $0.05 \mathrm{~s}$. We consider the coupling cases $\varepsilon=5.0,5.5,6.5$ and omit the first minute of data to allow for transients to decay.

In Fig. 2(b) we plot $\kappa$ as a function of $N$. Although the value of $\kappa$ depends on the details of the distribution of relative phases produced by the coupling, we see that $\kappa$ falls off as $1 / N$ with increasing $N$ for the homogeneous case, as expected.

\section{Vanishing coherence}

If we allow $c$ values below $1 / N$, or $c=0$, then the approximation becomes

$$
\left\langle x^{2}\right\rangle=\frac{1}{P} \frac{1}{P}\left(\sum_{i=1}^{N-1} \sum_{j=i+1}^{N} p_{i} p_{j}+p_{j} p_{i}\right),
$$

implying, in the absence of amplitude correlations, that

$$
\kappa=\frac{E\left(r^{2}\right)-E(r)^{2}}{E\left(r^{2}\right)} \approx 1-\left(\frac{7}{8}\right)^{2}=\frac{15}{64}=0.234 .
$$

However, for $c=0$ the Taylor expansion no longer converges because the $\left\langle x^{q}\right\rangle$ increase monotonically in magnitude as $q$ rises. In fact, when we perform the square root and then average over the distribution of possible $x$ values, we implicitly include cases with $|x|>1$, violating the condition for convergence. For $c \gg 1 / N$ these cases are sufficiently rare that they only dominate the $\left\langle x^{q}\right\rangle$ for very high $q$ and we can estimate $E(r)$ using the $q=2$ term only. For $c \approx 1 / N$ or less the state is essentially noninteracting and the value of $\kappa$ is near that for white noise $(0.215)$, which we evaluated above.

The addition of measurement noise to the mean field of a coherent ensemble obviously produces a mixed value $0<$ $\kappa<0.215$. The noise need not necessarily be white, of course. We can consider the general case of a noise signal that is stationary and reducible to a large number of independent Fourier components, each providing a small contribution to the signal. The same reasoning that we applied in Sec. III to find $\kappa$ for a large number of oscillations with independent phases shows that $\kappa$ for the noise will be the same as for white noise. For example, a detrended Brown noise signal gives $\kappa$ values indistinguishable from white noise. However, if the signal is contaminated by additive noise with a highly non-Gaussian distribution of absolute values, then this may systematically perturb $\kappa$ : For example, the $\kappa$ of a sinusoid is zero, so that adding a large sinusoid to another signal might produce a near zero $\kappa$. Thus it is desirable to filter out any such contamination prior to the analysis.

\section{An inhomogeneous case}

The simplest example of an inhomogeneous case is one in which the population can be broken down into $M$ subpopulations of size $p(m) N$, composed of individual oscillators that on average exhibit high coherence $c(m)$ with other members of their own subpopulation, but on average low coherence $c\left(m_{1}, m_{2}\right)$ with members of the other subpopulations, $\left\langle c\left(m_{1}, m_{2}\right)\right\rangle=c_{0}$. In this case it is convenient to reexpress the mean field as the sum of the mean fields of the individually coherent subpopulations, each of which has magnitude $p(m) N \sqrt{c(m)}$. We now effectively have $M$ oscillators instead of $N$. This gives

$$
E\left(r^{2}\right)=\frac{1}{N^{2}}\left(\sum_{m_{1}=1}^{M}\left[p\left(m_{1}\right) N\right]^{2} c\left(m_{1}\right)+2 \sum_{m_{1}=1}^{M-1} \sum_{m_{2}=m_{1}+1}^{M} p\left(m_{1}\right) N p\left(m_{2}\right) N c_{0}\right)
$$

and

$E(r)=\sqrt{E\left(r^{2}\right)}\left(1-\frac{1}{8\left[N^{2} E\left(r^{2}\right)\right]^{2}}\left\langle 2 \sum_{m_{1}=1}^{M-1} \sum_{m_{2}=m_{1}+1}^{M} p\left(m_{1}\right) N p\left(m_{2}\right) N\left[c\left(m_{1}, m_{2}\right)-c_{0}\right] 2 \sum_{m_{3}=1}^{M-1} \sum_{m_{4}=m_{3}+1}^{M} p\left(m_{3}\right) N p\left(m_{4}\right) N\left[c\left(m_{3}, m_{4}\right)-c_{0}\right]\right)\right.$.

We can group terms as before and assume that $\left\langle c\left(m_{1}, m_{2}\right) c\left(m_{3}, m_{4}\right)\right\rangle=c_{0}^{2}$ for four different sub-populations indexed $m_{1}, m_{2}, m_{3}, m_{4}$. The leading order $M^{3}$ non-vanishing terms in the numerator of $\left\langle x^{2}\right\rangle$ are of the $m_{1}=m_{3}, m_{1}=m_{4}, m_{2}=m_{3}$ or $m_{2}=m_{4}$ type, similarly to the homogenous case. 
If $M$ is very large then we return to the coherent case for $c_{0}>0$ with $\kappa$ tending to 0 ; where the number of subpopulations is not very large, however, the $M^{3}$ term in the numerator of $\left\langle x^{2}\right\rangle$ does not vanish and $\kappa$ is then intermediate between 0 and 0.215 . If we compare the behavior of several differentsized ensembles made up of different numbers (different values of $M$ ) of equivalent subpopulations (maintaining the same internal and cross coherence) then $p_{m}=1 / M$ and the $\left\langle x^{2}\right\rangle$ for the different ensembles will be expected to be proportional to $1 / M$, just as in the homogeneous case where it was proportional to $1 / N$.

Where there are more complex relationships $\left[c\left(m_{1}, m_{2}\right)\right.$ not purely real], the Taylor series approach may break down altogether, e.g., in the case of two subpopulations maintaining an antiphase relationship [negative $c(1,2)$ ]. See also the discussion below in Sec. V B.

\section{COMPLEX COHERENCE, CORRELATED AMPLITUDES, AND INTERMITTENCY $\kappa>0.215$}

In addition to the low- $\kappa$ phase-coupled ensembles described above, there are a number of interacting cases in which the ensemble yields $\kappa$ values even higher than those for the noninteracting case. The spread of possible mean-field values arising from such interacting ensembles is actually greater than that obtained when the phase distribution is entirely random.

\section{A. Harmonics}

A coherent state with higher harmonics, i.e., synchronized nonsinusoidal oscillations, will also produce a nonconstant mean field and intermediate values of $\kappa$. We can represent the mean field of a nonsinusoidal oscillation by the sum of harmonics $\sum_{n} a_{n} e^{i n / 2 \pi}$. Unlike the homogeneous coherent cases considered thus far, the power of the $n$th harmonic or oscillator will not necessarily tend to zero as a proportion of the total power as more harmonics are added (and the sum of the terms converges to the waveform) so $0<\kappa<1$. For a square wave $\kappa=0.1135$. Coherent harmonics can be reliably detected in noisy broadband signals [30].

\section{B. Complex coherence}

Consider general complex coherence $\left[c\left(m_{1}, m_{2}\right)\right.$ complex, conjugated by an exchange of subpopulations $m_{1}$ and $m_{2}$ ]. Where subpopulations making up a large fraction of the ensemble maintain a systematic phase difference relative to each other, the variance is in general larger than would be expected for a coherent ensemble with the same average mean field. For example, an ensemble composed of two otherwise equivalent subpopulations maintaining an antiphase relationship would have a vanishingly small mean field subject to the variability in both populations and consequently a relatively high $\kappa$ in the range $0.215<\kappa<1$.

\section{Correlated amplitudes}

If $\left\langle a_{i}^{2} a_{j}^{2}\right\rangle>p_{i} p_{j}, \quad\left\langle a_{i}^{2} a_{j} a_{l}\right\rangle>p_{i}\left\langle a_{j}\right\rangle\left\langle a_{l}\right\rangle, \quad$ and/or $\left.\left\langle a_{i} a_{j}^{2} a_{k}\right\rangle\right\rangle\left\langle a_{i}\right\rangle p_{j}\left\langle a_{k}\right\rangle$, then, due to correlations between $a_{j}(t)$ and $a_{i}(t)$, the assumptions made in Eq. (15) and thereafter break down and $\left\langle x^{2}\right\rangle$ in Eq. (32) increases. The
Taylor series approximation breaks down if the amplitude correlations become strong because the typical $|x|$ then approaches or exceeds unity.

Intuitively, we can see that correlated changes in amplitude may produce arbitrary changes in the mean field, with or without phase coherence. Even the mean field of random phasors will change proportionately if the amplitude of every phasor changes simultaneously. Thus $\kappa$ may in principle take any value within $0<\kappa<1$ if sufficiently strong amplitude variability and correlations are present.

\section{Intermittency}

We consider a simple multistable state in which the ensemble undergoes transitions between states with different mean-field strengths, e.g., two states with differing coherence. If the mean field of the less coherent state (state 1) is some proportion $\epsilon$ of the mean field in the more coherent state (state 2 ) and the proportion of time spent in the higher mean-field state is $\tau$, then we can write $E\left(r_{1}\right)=\epsilon E\left(r_{2}\right), E(r)=\tau E\left(r_{2}\right)+$ $(1-\tau) \epsilon E\left(r_{2}\right), \quad$ and $E\left(r^{2}\right) \approx \tau E\left(r_{2}^{2}\right)+(1-\tau) \epsilon^{2} E\left(r_{2}^{2}\right)$, giving

$$
\kappa \approx 1-\frac{[\tau+(1-\tau) \epsilon][\tau+(1-\tau) \epsilon]}{\tau+(1-\tau) \epsilon^{2}} .
$$

Here we assume that both states are sufficiently coherent to approximate $E\left(r_{i}^{2}\right)$ by $E\left(r_{i}\right)^{2}$ for both $i=1$ and 2 . In other words, we neglect the fluctuations within a state as they make a small contribution to the mean-field variance compared to the transitions between states. If $\epsilon$ goes to 1 , then $\kappa$ goes back to zero as expected for complete coherence but, for small $\epsilon$,

$$
\kappa \approx 1-\tau
$$

Thus, where the variability attributable to differences in meanfield strength of the two states is larger than the variability of either state, this is dominant and $\kappa=1-\tau$ for $\tau \gg \epsilon$. We see that a system making transitions between two states with low $\kappa$ can have a $\kappa$ value greater than that for white noise.

To model the intermittency we again use the classical (noiseless) Kuramoto ensemble of Eq. (13), but with timedependent coupling $\varepsilon(t)$, which is periodically pulsed between two values such that at each instant in time every oscillator in the ensemble is subjected to exactly the same value of $\varepsilon(t)$. We choose $\varepsilon(t)$ to have a value well above the critical coupling for some fraction of time less than the maximum pulse length of $60 \mathrm{~s}$; for the rest of the time, $\varepsilon(t)$ is well below the critical coupling. This procedure is designed to switch the entire ensemble between the synchronized and desynchronized states. For a signal length of $20 \mathrm{~min}$ and $\varepsilon(t)$ held high for durations of 5-55 s, the results shown in Fig. 3 confirm that the smaller the proportion of time spent in the synchronized state, the higher $\kappa$ becomes, tending towards unity for small $\tau$. For this model if $\tau<0.78$ then $\kappa>0.215$. Hence $\kappa$ can be larger than for the white noise case.

\section{THE MFVI OF EEG SIGNALS}

We further explore models relating to brain dynamics and intermittency, as discussed in Ref. [31]. We have investigated 


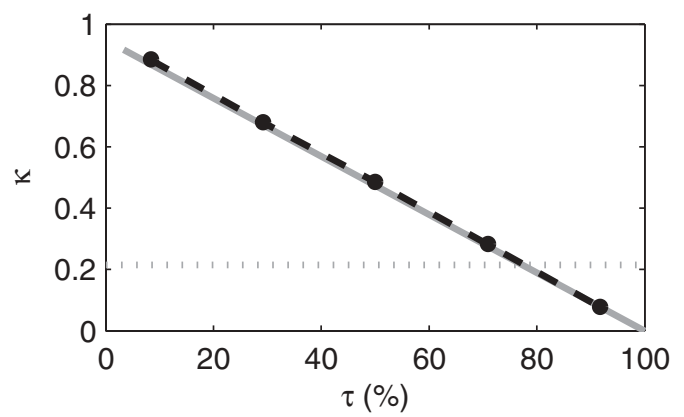

FIG. 3. Value of $\kappa$ (black filled circles) as a function of intermittency $\tau$. Results were obtained using a 2000-oscillator Kuramoto model, $D=0$, a Gaussian distribution of frequencies with width $\gamma=4.0$, and coupling strengths 20 and 0.1 . Each point was generated from a 20 -min time series. The $\tau$ value runs from $5 / 60$ to $55 / 60 \mathrm{~s}$. The simulations are in good agreement with the approximation (37) for $\kappa$ (slanted gray dashed line), where $\epsilon$ is estimated to range from 0.02 to 0.98 .

the changes in $\kappa$ that occur in two nonstandard states of an otherwise healthy human brain: anesthesia and an epileptic fit.

It is frequency synchrony between neurons that produces the spectral peaks in the EEG as shown in Fig. 4(a). We wish to know if the mean-field oscillations in these bands also exhibit evidence for phase or amplitude synchrony. A Hilbert transform of the EEG voltage time-series signal can be used to obtain the mean field or instantaneous signal amplitude $r$ at each point in time, from which we can find $\kappa=\operatorname{var}[g(r)] / \operatorname{mean}\left[g\left(r^{2}\right)\right]$.

\section{A. The MFVI for anesthesia vs normal wakefulness}

We have analyzed a typical pair of 20-min single-channel EEG time series recorded from a surgical patient participating in the BRACCIA research program. One time series was recorded from the awake patient and the other from the same patient after induction of general anesthesia. Finite impulse response (FIR) filters were used to subdivide the data into the following frequency intervals: $\delta(0.8-4 \mathrm{~Hz}), \theta(4-7.5 \mathrm{~Hz})$, $\alpha(7.5-14 \mathrm{~Hz}), \beta(14-22 \mathrm{~Hz}), \gamma(22-40 \mathrm{~Hz})$. Note that the precise selection of the frequency intervals is important in enabling meaningful conclusions to be drawn from the data. Each filtered time series was split into $30 \mathrm{~s}$ windows and a value of $\kappa$ was calculated for each window in each band. Additionally 20-min time series of white noise were filtered in the same way to show that the filtering does not significantly perturb the EEG results; unfiltered white noise gives $\kappa=0.2146 \pm 0.0007$. The $\kappa$ values for bandpass-filtered EEG signals and white noise are shown in Fig. 4(b).

A Wilcoxon test was used to evaluate the statistical significance of the $\kappa$ values. The distribution of mean $\kappa$ values obtained was also compared to time series of random noise with identical filtering. Unpaired tests of awake vs anesthetized show statistically significant differences in all bands apart from $\theta$ where $p=0.3998$. The test for $\alpha$ gives $p=0.0085$ and all the other bands have much lower values of $p$. Unpaired tests of awake compared to noise show statistically significant differences in all bands, with $p=0.04$ in the $\gamma$-band and
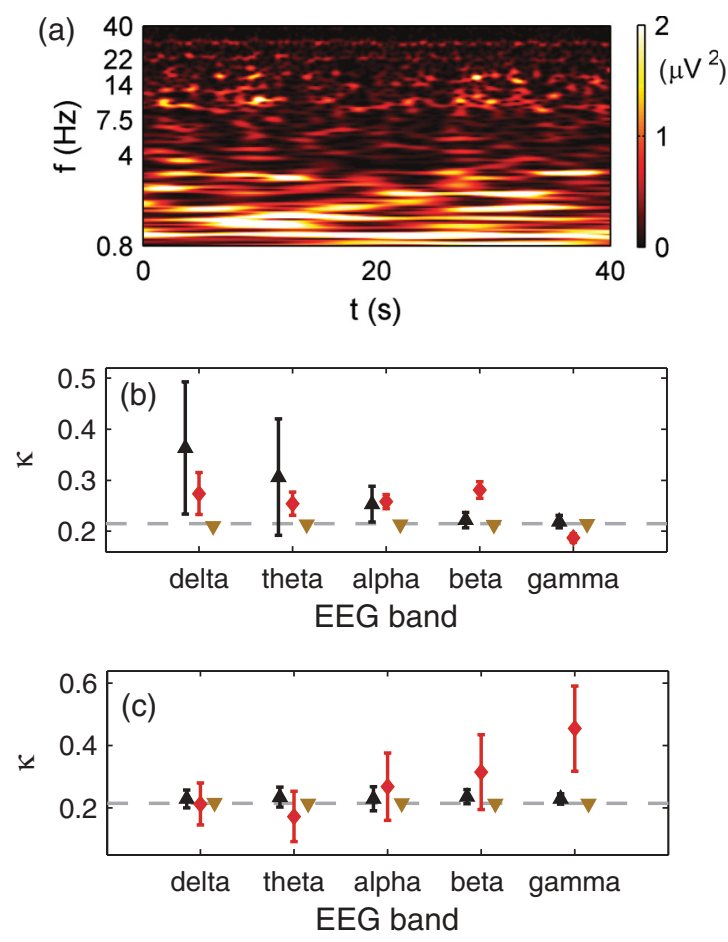

FIG. 4. (Color online) Applications to anesthesia and epilepsy. (a) Wavelet transform power of the $\delta-\gamma$ bands $(0.8-40 \mathrm{~Hz})$ in an EEG signal from a typical anesthetized subject shows epochs of frequency synchronization (yellow, across whole spectrum). (b) $\kappa$ values for single-channel EEG data (duration 20 mins, windowed into $30 \mathrm{~s}$ segments) from a typical subject, both anesthetized (red lozenges) and awake (black point-up triangles). Note that the $\kappa$ values for these two states are clearly distinguishable from white noise (khaki point-down triangles) and between themselves in certain EEG bands. (c) $\kappa$ values for EEG data from an epilepsy study [32]. The EEG from patients in their non-seizure state (black, point-up triangles) looks similar to white noise values (khaki point-down triangles), but it is still statistically different. During seizure (red lozenges), however, there is evidence for phase synchronization in the $\delta$ and especially $\theta$ bands and for intermittent and/or amplitude synchronization in the $\beta$ and $\gamma$ bands. In (b) and (c) the error bars refer to a one-standard-deviation spread in the values computed within different windows; they are omitted where the uncertainty is comparable with, or smaller than, the size of the symbol. In each case the horizontal grey dashed line indicates the value of $\kappa=0.215$ that would be seen in the absence of inter-oscillator interactions.

$p<0.0005$ for all other bands. Unpaired tests of anesthetized vs noise show significant differences $\left(p<10^{-10}\right)$ in all bands.

The results are inconsistent with either a non-interacting phase-desynchronized case, a fully phase-synchronized case, or continuous partial phase coherence. They suggest either (possibly intermittent) amplitude synchrony, or intermittent phase coherence, or both. The discrete time-separated peaks in the whole spectrum of the wavelet transform shown in Fig. 4(a) suggest intermittent synchronization, where different intermittencies [see Figs. 3 and 4(a)] lead to different values of $\kappa$ [see Figs. 3 and 4(b)].

We can compare the actual $\kappa$ values obtained to those of artificially generated surrogate signals with otherwise similar characteristics. Surrogates that preserve the actual distribution 
of mean-field values in the signal trivially preserve $\kappa$. A Fourier phase-shuffled surrogate [33], which preserves the Fourier spectrum of a signal but not the distribution, fails to preserve the high- $\kappa$ value obtained for an intermittently coupled ensemble or for a real-world EEG signal. The $\kappa$ values tend to be "whitened" in such surrogates, as a sum of many independent Fourier components with different amplitudes. Hence the $\kappa$ values are independent of the spectral characteristics of the signals.

\section{B. The MFVI in an epileptic fit vs normal wakefulness}

We also applied the measure to EEG data recorded for subjects in epileptic seizure, taken from Ref. [32] [see Fig. 4(c)]. Unpaired Wilcoxon tests were used to evaluate the statistical significance of the $\kappa$ values; $\kappa$ was calculated for 100 signals of length $23.6 \mathrm{~s}$. The distribution of mean $\kappa$ values obtained for all 100 signals was compared to 100 noise cases with identical filtering. The results were that: (i) for non-seizure vs seizure all are statistically different except in the case of the $\alpha$-band ( $p=0.426$ ), while for $\delta$ they are borderline ( $p=0.045$ ); and (ii) for non-seizure vs noise and seizure vs noise, they are all statistically different except for the $\delta$-band during seizure ( $p=0.4340)$.

Although the results for the subjects when awake are similar to those from the BRACCIA cohort $(\kappa>0.215)$, we see that for the $\theta$ band during seizure, $\kappa$ is actually less than for white noise, indicating pathological phase synchronization of oscillations in this band. The $\theta$ rhythm is commonly associated with the hippocampal formation and the hippocampal formation is associated with temporal lobe epilepsy. We speculate that this is why we observe phase synchronization of oscillations in this band during epileptic seizures. Furthermore, the $\beta$ and $\gamma$ bands (cognitive bands) show more $\kappa$ greater than in the awake case indicating more intermittency (i.e., longer epochs of the desynchronized state) and/or more amplitude synchronization [34].

\section{CONCLUSION}

The mean-field strength of an oscillator ensemble with random phases has highly predictable statistics. Interactions between the oscillators change the distribution of mean-field values in characteristic ways. Their mean-field variability index $\kappa$ is a dimensionless parameter that quantifies the distribution of mean-field values. Different $\kappa$ values arise
TABLE I. Characteristics of oscillator ensembles corresponding to different values of their mean-field variability index (MFVI) $\kappa$. It is assumed that any harmonics present in the raw signal have been removed by filtration.

\begin{tabular}{ll}
\hline \hline MFVI & \multicolumn{1}{c}{ State of the ensemble } \\
\hline$\kappa=0$ & complete phase synchronization \\
$0<\kappa<0.215$ & partially phase synchronized \\
$\kappa=0.215$ & noninteracting (or white noise) \\
$0.215<\kappa<1.000$ & complex coherence, correlated \\
& amplitudes, or intermittency \\
\hline \hline
\end{tabular}

depending on the coupling between the oscillators in an ensemble, but independent of spectral content or filtering, as summarized in Table I. There are three important caveats. First, the method only works for cases where we have good $a$ priori reason to believe that the signal is the summed output of many oscillators, each making a small contribution to the total: Otherwise, we cannot expect to deduce anything from the distribution of field values. Second, the method is best applied in conjunction with established approaches in order to gain deeper insight. Thus it can be combined with, for example, the wavelet transform, or with other time-frequency analysis and decomposition methods, to isolate particular spectral features whose $\kappa$ values can then be checked. Third, it is important to check for harmonics in the signal [30] and, if necessary, to filter these out because harmonics in a coherent system can push $\kappa$ above 0.215 .

We can distinguish coupled from uncoupled ensembles in the real world, using only the parameter $\kappa$ and irrespective of any other information but a series of independent measurements of their mean field $r$. In the case of EEG signals, in addition to the frequency synchronization evident from the wavelet spectrum, we find evidence for interactions such as intermittent phase coupling or amplitude coupling. It is evident that detailed applications of these ideas to, for example, the physiology of anesthesia or epilepsy, as well as to other aspects of brain function, are likely to be rewarding.

\section{ACKNOWLEDGMENTS}

This work was supported by the Engineering and Physical Sciences Research Council (United Kingdom) (Grant No. EP/100999X1) and by the European Commission FP7 program BRACCIA [Grant No. 51733 (NEST)].
[1] S. H. Strogatz, Sync: The Emerging Science of Spontaneous Order (Hyperion, New York, 2003).

[2] A. Koseska, E. Ullner, E. Volkov, J. Kurths, and J. Garcia-Ojalvo, J. Theor. Biol. 263, 189 (2010).

[3] O. Mondragon-Palomino, T. Danino, J. Selimkhanov, L. Tsimring, and J. Hasty, Science 333, 1315 (2011).

[4] Y. Kuramoto, Chemical Oscillations, Waves, and Turbulence (Springer-Verlag, Berlin, 1984).

[5] J. A. Acebrón, L. L. Bonilla, C. J. Pérez Vicente, F. Ritort, and R. Spigler, Rev. Mod. Phys. 77, 137 (2005).

[6] S. H. Strogatz, Nature (London) 410, 268 (2001).
[7] T. D. Frank, A. Daffertshofer, C. E. Peper, P. J. Beek, and H. Haken, Physica D 144, 62 (2000).

[8] J. H. Sheeba, A. Stefanovska, and P. V. E. McClintock, Biophys. J. 95, 2722 (2008).

[9] G. Deco, V. Jirsa, A. R. McIntosh, O. Sporns, and R. Kötter, Proc. Natl. Acad. Sci. USA 106, 10302 (2009).

[10] M. Breakspear, S. Heitmann, and A. Daffertshofer, Frontiers Human Neurosci. 4, 190 (2010).

[11] J. Cabral, E. Hugues, O. Sporns, and G. Deco, NeuroImage 57, 130 (2011).

[12] A. Stefanovska, IEEE Eng. Med. Bio. Mag. 26, 25 (2007). 
[13] A. Bahraminasab, F. Ghasemi, A. Stefanovska, P. V. E. McClintock, and R. Friedrich, New J. Phys. 11, 103051 (2009).

[14] B. Musizza, F. Bajrović, J. Petrovčič, A. Stefanovska, and S. Ribarič, Fluct. Noise Lett. 11, 1240018 (2012).

[15] B. Musizza, A. Stefanovska, P. V. E. McClintock, M. Paluš, J. Petrovčič, S. Ribarič, and F. F. Bajrović, J. Physiol. (London) 580, 315 (2007).

[16] G. Buzsáki, Rhythms of the Brain (Oxford University Press, Oxford, 2006).

[17] P. E. McSharry, L. A. Smith, and L. Tarassenko, Nat. Med. 9, 241 (2003).

[18] A. Pikovsky and M. Rosenblum, Physica D 238, 27 (2009).

[19] A. Pikovsky and M. Rosenblum, Physica D 240, 872 (2011).

[20] R. Suresh, D. V. Senthilkumar, M. Lakshmanan, and J. Kurths, Phys. Rev. E 86, 016212 (2012).

[21] C. A. S. Batista, E. L. Lameu, A. M. Batista, S. R. Lopes, T. Pereira, G. Zamora-Lopez, J. Kurths, and R. L. Viana, Phys. Rev. E 86, 016211 (2012).

[22] S. Petkoski and A. Stefanovska, Phys. Rev. E 86, 046212 (2012).

[23] A. A. Temirbayev, Z. Z. Zhanabaev, S. B. Tarasov, V. I. Ponomarenko, and M. Rosenblum, Phys. Rev. E 85, 015204 (2012).

[24] Y. Shiogai, A. Stefanovska, and P. V. E. McClintock, Phys. Rep. 488, 51 (2010)
[25] A. Pikovsky, M. Rosenblum, and J. Kurths, SynchronizationA Universal Concept in Nonlinear Sciences (Cambridge University Press, Cambridge, 2001).

[26] D. Rudrauf, A. Douiri, C. Kovach, J. P. Lachaux, D. Cosmelli, M. Chavez, C. Adam, B. Renault, J. Martinerie, and M. L. Van Quyen, Neuroimage 31, 209 (2006).

[27] B. Kralemann, A. Pikovsky, and M. Rosenblum, Chaos 21, 025104 (2011).

[28] J. W. Strutt, Scientific Papers by John William Strutt, Baron Rayleigh (Cambridge University Press, Cambridge, 1902), Vol. 3, pp. 47-187 (see in particular p. 52).

[29] A. Bandrivskyy, A. Bernjak, P. V. E. McClintock, and A. Stefanovska, Cardiovasc. Eng. 4, 89 (2004).

[30] L. W. Sheppard, A. Stefanovska, and P. V. E. McClintock, Phys. Rev. E 83, 016206 (2011).

[31] A. C. Hale, T. Hansard, L. W. Sheppard, P. V. E. McClintock, and A. Stefanovska, Fluct. Noise Lett. 11, 1240011 (2012).

[32] R. G. Andrzejak, K. Lehnertz, F. Mormann, C. Rieke, P. David, and C. E. Elger, Phys. Rev. E 64, 061907 (2001).

[33] T. Schreiber and A. Schmitz, Physica D 142, 346 (2000).

[34] The control signals in the epilepsy data were sliced to remove all artifacts. This was not done to the BRACCIA awake data, whence its slightly higher $\kappa$ values. 